\title{
Not all anemia is solely due to iron deficiency
}

\author{
Julian Rössler, Alexander Kaserer, Gabriela H. Spahn, Donat R. Spahn \\ Institute of Anesthesiology, University of Zurich and University Hospital Zurich, Zurich, Switzerland \\ Correspondence to: Prof. Dr. med. Donat R. Spahn, FRCA. Institute of Anaesthesiology, University Hospital Zurich, Raemistrasse 100, 8091 Zurich, \\ Switzerland. Email: donat.spahn@usz.ch. \\ Provenance and Peer Review: This is an invited article commissioned and reviewed by the Section Editor Laura Chiara Guglielmetti (Cantonal \\ Hospital Winterthur, Kantonsspital Winterthur, Winterthur, Switzerland). \\ Response to: Heinrich S, Loop T. Preoperative treatment of anemia-could an ultra-short-term multimodal approach be beneficial for patients \\ undergoing lung surgery? J Thorac Dis 2019;11:S1913-5.
}

Submitted Dec 09, 2019. Accepted for publication Dec 29, 2019.

doi: $10.21037 /$ jtd.2019.12.129

View this article at: http://dx.doi.org/10.21037/jtd.2019.12.129

Heinrich and Loop (1) recently commented our study on the ultra-short term combination treatment of anemia and iron deficiency in patients undergoing cardiac surgery (2). They discussed whether such treatment might be an option in lung cancer surgery and came to the conclusion that the optimal approach in lung cancer surgery would be to treat preoperative anemia (without specifying by which means) and to administer intravenous iron in patients with a higher risk of severe postoperative anemia such as following neo-adjuvant chemotherapy or pneumonectomy. In their imagination, this makes sense, since they initially state "iron deficiency seems to be the commonest cause of anemia in the surgical population". Two things are important to note in regard to their argument: First, their reference of Klein et al., is not at all about iron deficiency but on the impact of anemia prior to cardiac surgery (3). Secondly, the assumption that because iron deficiency is the most common cause of anemia in the surgical population and cancer patients are often anemic, iron deficiency must also be the most common cause in cancer patients, is a syllogistic fallacy.

On a world-wide basis, iron deficiency anemia is indeed the most prevalent type of anemia (4). In surgical populations iron deficiency anemia as well as isolated iron deficiency are associated with increased morbidity and mortality $(5,6)$. However, not all anemia is solely due to iron deficiency. In patients with cancer, the most common type of anemia is anemia of inflammation as recently described by Weiss et al. (7). The main mechanisms that finally result in anemia in this type of anemia are: (I) iron deficiency or reduced iron availability resulting in iron-restricted erythropoiesis, (II) reduced erythropoietin response to anemia, (III) reduced erythroid cell differentiation upon erythropoietin stimulation and (IV) a shortened red blood cell life span. Since iron availability is reduced and at the same time the erythropoietin response and erythropoietin efficacy are blunted, Weiss et al. propose a "combination of iron therapy and erythropoiesis-stimulating agents" in such patients (7). Combining intravenous iron and subcutaneous erythropoietin thus may not only be an option but maybe the key to success in patients with anemia of inflammation.

Treating preoperative anemia in patients undergoing lung cancer surgery is important since anemia has repeatedly been shown to be a major risk factor for mortality (8) and red cell transfusions (8) which additionally increase mortality (9). Obviously, the only way out is the treatment of preoperative anemia and erythropoietin will often be part of such treatment (7). Unfortunately, there are concerns regarding the administration of erythropoietin with a number of physicians. However, preoperatively we are administering a single dose of erythropoietin and in numerous meta-analyses preoperative use of erythropoietin has been shown to be highly efficacious and not associated with side effects (10-12): in the perioperative setting, erythropoiesis-stimulating agents successfully reduce blood transfusions without increasing the risk of 45-day mortality, acute myocardial infarction, bowel ischemia, acute kidney injury or thromboembolic events (11). In the critical care setting, a meta-analysis of close to 1 million patients found the off-label use of erythropoiesis-stimulating agents to 
be associated with a reduction mortality by a risk ratio of 0.76 (95\% CI: 0.61 to 0.92 ). The same meta-analysis found no evidence of increased myocardial infarction, stroke, venous thromboembolism or any adverse events (12). While randomized trials are commonly not powered to adequately investigate safety profiles, these meta-analyses provide evidence on the safety and benefits of erythropoiesisstimulating agents in the perioperative setting and in the critically ill patient.

Heinrich and Loop further question the transferability of a combination anemia treatment into lung cancer surgery. They doubt the necessity of such a treatment in light of an often 28-day long surgery preparation interval (1). While our study was a pragmatic approach to often time sensitive cardiac procedures, a longer preparation interval may actually improve patient outcomes by allowing more time for preoperative hemopoietic recovery. In this setting, anemia treatment may be tailored to the cause of anemia. Here however, erythropoiesis-stimulating agents are often also necessary, if e.g. anemia of inflammation is present (7).

In our original study, we analyzed the acquisition costs of red blood cells and combination drug treatment: Total costs were higher in the treatment (mean of 1,052 \pm 674 Swiss Francs) than in the placebo group (mean of $480 \pm 704$ Swiss Francs) (2). In light of the total acquisition costs, Heinrich and Loop relinquish an U.S. Food and Drug Administration container label extension for red blood cell units that states the following contraindication: "Red-cell-containing components should not be used to treat anemias that can be corrected with specific hematinic medications such as iron, vitamin B12, folic acid, or erytbropoietin." (13). While resources may be sparse in current healthcare systems, we would advise to not neglect evidence-based recommendations for a mean saving of 572 Swiss Francs. Further, our study only estimated pure acquisition cost of a combination treatment or red blood cells. The systemic financial burden of blood transfusions is difficult to calculate, but has been estimated that transfused patients have a propensity matched mean higher total costs of $\$ 1,777 \pm 36$ per admission (14).

In a pragmatic way, patients undergoing major surgery resulting a red blood cell transfusion rate of $\geq 10 \%$ or with an expected blood loss of $\geq 500 \mathrm{~mL}$ need to be tested for hemoglobin concentration, ferritin, transferrin saturation, creatinine and C-reactive protein. With these simple laboratory data anemia and iron deficiency can be detected, the anemia broadly categorized and treated according to an algorithm. How this algorithm looks like in detail may be left to individual hospitals and incorporate the local drug availability which varies significantly world-wide.

\section{Acknowledgments}

Funding: None.

\section{Footnote}

Conflicts of Interest: Dr. A Kaserer received honoraria/travel support for lecturing from Bayer AG, Zürich, Switzerland. Dr. DR Spahn's academic department is receiving grant support from the Swiss National Science Foundation, Berne, Switzerland, the Swiss Society of Anesthesiology and Reanimation (SGAR), Berne, Switzerland, the Swiss Foundation for Anesthesia Research, Zurich, Switzerland, Vifor SA, Villars-sur-Glâne, Switzerland. Dr. DR Spahn is co-chair of the ABC-Trauma Faculty, sponsored by unrestricted educational grants from Novo Nordisk Health Care AG, Zurich, Switzerland, CSL Behring GmbH, Marburg, Germany, LFB Biomédicaments, Courtaboeuf Cedex, France and Octapharma AG, Lachen, Switzerland. Dr. DR Spahn received honoraria/travel support for consulting or lecturing from: Danube University of Krems, Austria, US Department of Defense, Washington, USA, European Society of Anesthesiology, Brussels, BE, Korean Society for Patient Blood Management, Seoul, Korea, Korean Society of Anesthesiologists, Seoul, Korea, Baxter AG, Volketswil, Switzerland, Baxter S.p.A., Roma, Italy, Bayer AG, Zürich, Switzerland, Bayer Pharma AG, Berlin, Germany, B. Braun Melsungen AG, Melsungen, Germany, Boehringer Ingelheim GmbH, Basel, Switzerland, BristolMyers-Squibb, Rueil-Malmaison Cedex, France and Baar, Switzerland, CSL Behring GmbH, Hattersheim am Main, Germany and Berne, Switzerland, Celgene International II Sàrl, Couvet, Switzerland, Curacyte AG, Munich, Germany, Daiichi Sankyo AG, Thalwil, Switzerland, GlaxoSmithKline GmbH \& Co. KG, Hamburg, Germany, Haemonetics, Braintree, MA, USA, Instrumentation Laboratory (Werfen), Bedford, MA, USA, LFB Biomédicaments, Courtaboeuf Cedex, France, Merck Sharp \& Dohme, Kenilworth, New Jersey, USA, Octapharma AG, Lachen, Switzerland, Organon AG, Pfäffikon/SZ, Switzerland, PAION Deutschland GmbH, Aachen, Germany, Pharmacosmos A/ S, Holbaek, Denmark, Photonics Healthcare B.V., Utrecht, Netherlands, Pierre Fabre Pharma, Alschwil, Switzerland, Roche Diagnostics International Ltd, Reinach, Switzerland, Roche Pharma AG, Reinach, Switzerland, Sarstedt AG \& Co., Sevelen, Switzerland and Nümbrecht, Germany, 
Schering-Plough International, Inc., Kenilworth, New Jersey, USA, Tem International GmbH, Munich, Germany, Verum Diagnostica GmbH, Munich, Germany, Vifor Pharma, Munich, Germany, Vienna, Austria and Villarssur-Glâne, Switzerland, Vifor (International) AG, St. Gallen, Switzerland, Zuellig Pharma Holdings, Singapore, Singapore. The other authors have no conflicts of interest to declare.

Ethical Statement: The authors are accountable for all aspects of the work in ensuring that questions related to the accuracy or integrity of any part of the work are appropriately investigated and resolved.

Open Access Statement: This is an Open Access article distributed in accordance with the Creative Commons Attribution-NonCommercial-NoDerivs 4.0 International License (CC BY-NC-ND 4.0), which permits the noncommercial replication and distribution of the article with the strict proviso that no changes or edits are made and the original work is properly cited (including links to both the formal publication through the relevant DOI and the license). See: https://creativecommons.org/licenses/by-nc-nd/4.0/.

\section{References}

1. Heinrich S, Loop T. Preoperative treatment of anemiacould an ultra-short-term multimodal approach be beneficial for patients undergoing lung surgery? J Thorac Dis 2019;11:S1913-5.

2. Spahn DR, Schoenrath F, Spahn GH, et al. Effect of ultrashort-term treatment of patients with iron deficiency or anaemia undergoing cardiac surgery: a prospective randomised trial. Lancet 2019;393:2201-12.

3. Klein AA, Collier TJ, Brar MS, et al. The incidence and importance of anaemia in patients undergoing cardiac surgery in the UK - the first Association of Cardiothoracic Anaesthetists national audit. Anaesthesia 2016;71:627-35.

4. Kassebaum NJ, Collaborators GBDA. The Global

Cite this article as: Rössler J, Kaserer A, Spahn GH, Spahn DR. Not all anemia is solely due to iron deficiency. J Thorac Dis 2020;12(3):1130-1132. doi: 10.21037/jtd.2019.12.129
Burden of Anemia. Hematol Oncol Clin North Am 2016;30:247-308.

5. Rössler J, Schoenrath F, Seifert B, et al. Iron deficiency is associated with higher mortality in patients undergoing cardiac surgery: a prospective study. Br J Anaesth 2020;124:25-34.

6. Musallam KM, Tamim HM, Richards T, et al. Preoperative anaemia and postoperative outcomes in non-cardiac surgery: a retrospective cohort study. Lancet 2011;378:1396-407.

7. Weiss G, Ganz T, Goodnough LT. Anemia of inflammation. Blood 2019;133:40-50.

8. Jean RA, DeLuzio MR, Kraev AI, et al. Analyzing Risk Factors for Morbidity and Mortality after Lung Resection for Lung Cancer Using the NSQIP Database. J Am Coll Surg 2016;222:992-1000.e1.

9. Wang T, Luo L, Huang H, et al. Perioperative Blood Transfusion Is Associated With Worse Clinical Outcomes in Resected Lung Cancer. Ann Thorac Surg 2014;97:1827-37.

10. Zhao F, Wang Y, Liu L, et al. Erythropoietin for cancerassociated malignant anemia: A meta-analysis. Mol Clin Oncol 2017;6:925-30.

11. Cho BC, Serini J, Zorrilla-Vaca A, et al. Impact of Preoperative Erythropoietin on Allogeneic Blood Transfusions in Surgical Patients: Results From a Systematic Review and Meta-analysis. Anesth Analg 2019;128:981-92.

12. Mesgarpour B, Heidinger BH, Roth D, et al. Harms of off-label erythropoiesis-stimulating agents for critically ill people. Cochrane Database Syst Rev 2017;8:CD010969.

13. AABB. Circular of Information for the Use of Human Blood and Blood Components 2017 [accessed 09. December 2019]. Available online: http://www.aabb.org/ tm/coi/Documents/coi1017.pdf

14. Klika AK, Small TJ, Saleh A, et al. Primary total knee arthroplasty allogenic transfusion trends, length of stay, and complications: nationwide inpatient sample 20002009. J Arthroplasty 2014;29:2070-7. 